\title{
Application of the THM to the investigation of reactions induced by unstable nuclei: the ${ }^{18} \mathbf{F}(\mathbf{p}, \alpha){ }^{15} \mathrm{O}$ case
}

\author{
Marco La Cognata ${ }^{1, *}$, Rosario G. Pizzone ${ }^{1}$, Jordi Josée ${ }^{2,3}$, Margarita Hernanz ${ }^{3,4}$, Silvio Cherubini ${ }^{1,5}$, Marisa Gulino ${ }^{1,6}$, \\ Giuseppe G. Rapisarda ${ }^{1,5}$, and Claudio Spitaleri ${ }^{1,5}$ \\ ${ }^{1}$ INFN - Laboratori Nazionali del Sud, Catania, Italy \\ ${ }^{2}$ Departament de Física, EEBE, Universitat Politècnica de Catalunya, 08019 Barcelona, Spain \\ ${ }^{3}$ Institut d'Estudis Espacials de Catalunya, 08034 Barcelona, Spain \\ ${ }^{4}$ Institut de Ciencies de l'Espai (ICE-CSIC), Campus UAB, c/ Can Magrans s/n, 08193 Bellaterra, Spain \\ ${ }^{5}$ Dipartimento di Fisica e Astronomia, Università degli Studi di Catania, Catania, Italy \\ ${ }^{6}$ Facoltà di Ingegneria ed Architettura, Kore University, Viale delle Olimpiadi 1, 94100 Enna, Italy
}

\begin{abstract}
The Trojan Horse Method is applied to the investigation of the ${ }^{18} \mathrm{~F}(\mathrm{p}, \alpha){ }^{15} \mathrm{O}$ reaction, by extracting the quasi free contribution to the ${ }^{2} \mathrm{H}\left({ }^{18} \mathrm{~F}, \alpha^{15} \mathrm{O}\right) n$ process. For the first time the method is applied to a reaction of astrophysical importance involving a radioactive nucleus. After investigating the reaction mechanism populating the $\alpha+{ }^{15} \mathrm{O}+n$ exit channel, we could extract the ${ }^{18} \mathrm{~F}(\mathrm{p}, \alpha){ }^{15} \mathrm{O}$ cross section and calculate the astrophysical factor over the $0-1 \mathrm{MeV}$ energy interval. The possibility of exploring the cross section with no need of extrapolation allowed us to to point out the possible occurrence of a $7 / 2^{+}$state at $126 \mathrm{keV}$, which would strongly influence the trend of the astrophysical factor at the energies of astrophysical interest. However, the low energy resolution prevents us to draw definite conclusions. Possible astrophysical consequences are also discussed, motivating further work on this reaction.
\end{abstract}

\section{$1{ }^{18} \mathrm{~F}$ in astrophysics}

Classical novae are among the most energetic thermonuclear explosions in the Cosmos, releasing about $10^{44} \mathrm{erg}$. They occur in a binary system made up of a white dwarf and a less evolved companion. If matter is transferred from the less evolved star to the compact companion, it accumulates under degenerate conditions until the thermonuclear runaway is ignited. In the envelope temperatures in excess of $10^{8} \mathrm{~K}$ are reached and nuclei undergo a sequence of proton captures producing $\beta$-unstable nuclei. Moreover, during these events about $10^{-3}-10^{-7} \mathrm{M}_{\odot}$ are ejected into the interstellar medium, enriched in $\mathrm{CNO}$ and intermediatemass elements such as $\mathrm{Ne}, \mathrm{Na}, \mathrm{Mg}, \mathrm{Al}$ (see [1-4] for recent reviews on classical novae).

The production of $\beta$-unstable nuclei suggests the possibility to observe both $\gamma$-ray emission due to the disintegration of short-lived radioactive species such as ${ }^{13} \mathrm{~N}$ and ${ }^{18} \mathrm{~F}$ and a late emission, due to medium-lived unstable nuclei such as ${ }^{7} \mathrm{Be}$ and ${ }^{22} \mathrm{Na}$, determining the production of definite $\gamma$-lines from the de-excitation of excited states of their daughter nuclei. However, while classical novae have been observed in all wavelengths, from radio-waves to $\gamma$ rays with energies in excess of $100 \mathrm{MeV}$, they have been quite elusive in the $\sim 0.1-10 \mathrm{MeV}$ range, where the contribution of $\gamma$-rays from radioactive nuclei is expected.

In detail, the $\gamma$-ray spectrum should be dominated by an emission at energies $\leq 511 \mathrm{keV}$, originated by the an-

*e-mail: lacognata@lns.infn.it nihilation of positrons from ${ }^{13} \mathrm{~N}$ and ${ }^{18} \mathrm{~F} \beta^{+}$decay. The major contribution is predicted to come from ${ }^{18} \mathrm{~F}$, owing to its lifetime $T_{1 / 2} \sim 110 \mathrm{~s}$. This would allows ${ }^{18} \mathrm{~F}$ to survive until the outer layers of the nova becomes transparent to $\gamma$-rays [3]. For this reason, the detection of the $\gamma$ lines from ${ }^{18} \mathrm{~F}$ would help constraining models. However, only upper limits on the ${ }^{18} \mathrm{~F}$ annihilation line have been set to date, leading to the establishment of a detectability distance, namely, the maximum distance of a classical nova for which the $\gamma$-line from ${ }^{18} \mathrm{~F}$ can be observed. At present, the most stringent detectability distance of the $511 \mathrm{keV}$ line has been set by the SPI spectrometer on board the space-borne $\gamma$-ray observatory INTEGRAL, $\sim 3 \mathrm{kpc}$ $[5,6]$.

Such constraint is strongly dependent on the predicted ${ }^{18} \mathrm{~F}$ abundances produced during novae outbursts. In turn, accurate modeling of the elemental yields are critically dependent on the cross sections of the production and destruction nuclear reactions entering ${ }^{18} \mathrm{~F}$ nucleosynthesis.

\section{Present status of the ${ }^{18} \mathrm{~F}(\mathbf{p}, \alpha){ }^{15} \mathrm{O}$ cross section measurements}

Production of ${ }^{18} \mathrm{~F}$ in classical novae starts with the ${ }^{16} \mathrm{O}(\mathrm{p}, \gamma){ }^{17} \mathrm{~F}$ reaction. Then, ${ }^{18} \mathrm{~F}$ is either produced by proton capture on ${ }^{17} \mathrm{~F}$, through the ${ }^{18} \mathrm{Ne}\left(\beta^{+}\right){ }^{18} \mathrm{~F}$ chain, or following ${ }^{17} \mathrm{~F} \beta^{+}$decay by proton capture on the daughter ${ }^{17} \mathrm{O}$ nucleus. Since the ${ }^{18} \mathrm{~F}$ half-life is quite large in comparison with the outburst timescale, it is primarily destroyed 
through proton-captures, the ${ }^{18} \mathrm{~F}(\mathrm{p}, \gamma){ }^{19} \mathrm{Ne}$ and, chiefly, the ${ }^{18} \mathrm{~F}(\mathrm{p}, \alpha){ }^{15} \mathrm{O}$ reaction, $\sim 1000$ times more efficient than the former [4]. However, the ${ }^{18} \mathrm{~F}(\mathrm{p}, \alpha){ }^{15} \mathrm{O}$ reaction is the most uncertain process in ${ }^{18} \mathrm{~F}$ production (and destruction) network, even if many direct and indirect measurements as well as theoretical studies (see, e.g. Ref.[7]) have focused on its investigation at astrophysical energies.

Direct measurements are very challenging because of the energy range of interest, below about $400 \mathrm{keV}$, where cross sections are vanishingly small, and because of the need of a radioactive beam, whose produced intensities seldom exceed $10^{6}$ ions per second. Direct measurements include the results of Ref.[8-13], focusing on the exploration of a broad $3 / 2^{+}$resonance at about $700 \mathrm{keV}$ in the center-of-mass system. A few measurements could reach astrophysical energies, measuring few points around $300 \mathrm{keV}$, where an additional resonance $\left(\mathrm{J}^{\pi}=3 / 2^{-}\right)$was observed $[14,15]$. However, owing to poor energy resolution and large uncertainties, such measurements, though extremely complicated, could not set strong constraint on the trend of the cross section or, equivalently, of the astrophysical factor. The trend of the cross section is indeed very important to calculate the reaction rate, that is the input parameter of astrophysical calculations. Therefore, R-matrix was often used to extrapolate and interpolate the trend of the astrophysical factor; however, the occurrence of interfering resonances made it impossible to establish the behaviour of the astrophysical factor with the accuracy requested by astrophysical models.

With this respect, indirect techniques proved to be very helpful in the search of resonances at astrophysical energies, by performing the spectroscopy of ${ }^{19} \mathrm{Ne}$ intermediate compound system or of its mirror nucleus ${ }^{19} \mathrm{~F}$. Among others, the $(d, p)$ reaction on ${ }^{18} \mathrm{~F}$ and the ${ }^{15} \mathrm{~N}-\alpha$ elastic scattering were used to explore the ${ }^{19} \mathrm{~F}$ spectrum at energies corresponding to those of astrophysical interest (see [16] and [17], respectively). Similarly, $p$ inelastic scattering off ${ }^{19} \mathrm{Ne}$ was used to perform its spectroscopy [18], as well as the $(d, n)$ transfer reaction on ${ }^{18} \mathrm{~F}$ [19] and the $(p, d)$ neutron pickup on ${ }^{20} \mathrm{Ne}[20,21]$. These studies and charge symmetry considerations show that low-energy swave resonances are extremely influential, in particular the $3 / 2^{+}$levels observed and/or predicted above and below the proton emission threshold (about $6.410 \mathrm{MeV}$ ) in ${ }^{19} \mathrm{Ne}$. This is due to the occurrence of a strong $3 / 2^{+}$ resonance at $E_{c . m}$. $=665 \mathrm{keV}$ in the ${ }^{18} \mathrm{~F}(\mathrm{p}, \alpha)^{15} \mathrm{O}$ astrophysical factor, possibly interfering with lower-lying $3 / 2^{+}$state. The consequent interference pattern would strongly influence the trend of the astrophysical factor well within the energy window of astrophysical importance. Since this is presently the largest source of uncertainty affecting the ${ }^{18} \mathrm{~F}(\mathrm{p}, \alpha)^{15} \mathrm{O}$ astrophysical factor, recently the ${ }^{19} \mathrm{~F}\left({ }^{3} \mathrm{He}, t \gamma\right){ }^{19} \mathrm{Ne}$ and the ${ }^{19} \mathrm{~F}\left({ }^{3} \mathrm{He}, t\right){ }^{19} \mathrm{Ne}$ reactions were studied aiming at determining the energies and proton widths of the predicted $3 / 2^{+}{ }^{19} \mathrm{Ne}$ states (see [22] and [23], respectively).

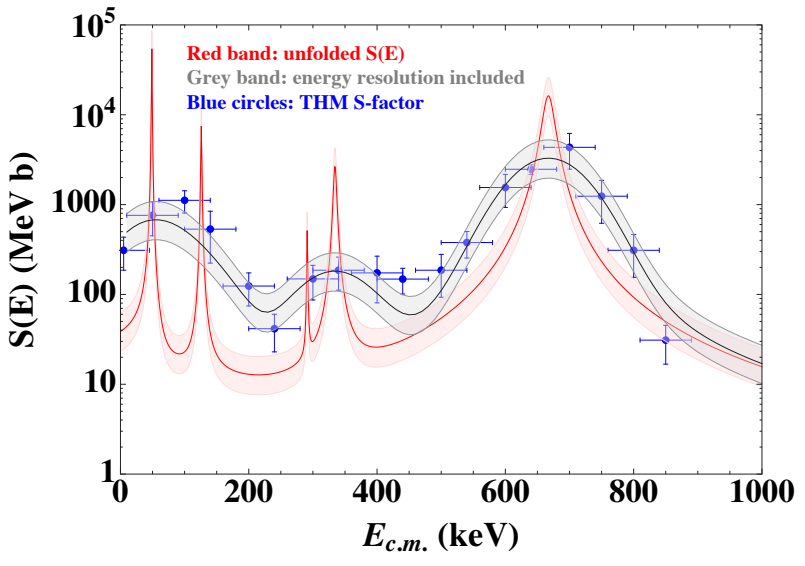

Figure 1. R-matrix analysis of the THM astrophysical factor (blu points), under the assumption of $\mathrm{J}^{\pi}=3 / 2^{+}$for the $6460 \mathrm{keV}{ }^{19} \mathrm{Ne}$ state as discussed in $[25,26]$. The black line is the smoothed Rmatrix calculation, accounting for a $53 \mathrm{keV}$ energy spread (standard deviation), with parameters given in table 1 . The red line is the corresponding deconvoluted astrophysical factor. The grey and the red bands show the uncertainty on the R-matrix analysis (standard deviation) due to the experimental errors affecting the THM S-factor.

\section{Indirect measurement of the ${ }^{18} \mathbf{F}(\mathbf{p}, \alpha){ }^{15} \mathbf{O}$ cross section using the THM}

Because of the ambiguities in the trend of the ${ }^{18} \mathrm{~F}(\mathrm{p}, \alpha){ }^{15} \mathrm{O}$ astrophysical factor at ultra-low energies, we decided the apply the Trojan Horse Method (THM) to its determination. Indeed, THM is an alternative and valuable approach to obtain the bare-nucleus cross section of a reaction induced by charged particles at energies lower than the Coulomb barrier, thanks to the use of suitable reactions with three particles in the exit channel. A recent review of the method is given in Ref.[24]. Here we recall that THM is based on the selection of the quasi-free reaction mechanism, namely, we single out the condition under which a cluster system (for instance, a deuteron), usually referred to as Trojan Horse nucleus, is used to transfer a participant cluster while the remaining cluster is emitted without influencing the participant-projectile interaction. In the case of the ${ }^{18} \mathrm{~F}(\mathrm{p}, \alpha){ }^{15} \mathrm{O}$ reaction, we studied the ${ }^{2} \mathrm{H}\left({ }^{18} \mathrm{~F}, \alpha^{15} \mathrm{O}\right) n$ three-body reaction, the neutron being the spectator to the nuclear reaction of astrophysical interest. Since the energy of the incident ${ }^{18} \mathrm{~F}$ is chosen large enough to overcome the Coulomb barrier of the ${ }^{18} \mathrm{~F}-d$ interaction, the deuteron breakup takes place inside the nuclear field, so that Coulomb repulsion is greatly suppressed. Such energies, of the order of several $\mathrm{AMeV}$, are usually much larger than those corresponding to the atomic degrees of freedom, so electron screening effects are also negligible. On the other hand, both the energy spent to break the Trojan Horse nucleus and intercluster motion make it possible to span the astrophysical energy region with a single beam energy.

Two experiments were carried out, one at CNSRIKEN (see [25] for more details) and one at the Texas A\&M University [26]. Results are in agreement with each 
other within uncertainties and their weighed average is shown as blue circles in figure 1. In the figure, vertical error bars account for statistical and normalization errors, while the horizontal error bar indicates the calculated energy resolution, strictly linked to the bin width used in the data analysis. More details can be found in the original works $[25,26]$. We then performed an R-matrix analysis of the THM data, starting from the work [20] and modifying the parameters in order to best reproduce the THM $\mathrm{S}$-factor. The adopted procedure is discussed at length in [27]. Here we remind that the best fit curve (shown as a black line in figure 1) is achieved by assuming the $(++)(++)$ interference pattern, following to the notation in Fig. 3 of [20], excluding the $7 \mathrm{keV}$ resonance corresponding to the $6.417 \mathrm{MeV}$ state in ${ }^{19} \mathrm{Ne}$ (see table 1) and inserting a $7 / 2^{+}$state of ${ }^{19} \mathrm{Ne}$ at $6.537 \mathrm{MeV}$, whose occurrence was already pointed out in $[25,26]$. Also, to compare the calculated astrophysical factor with the experimental THM one, the effect of the energy resolution is taken into account, by smearing the theoretical curve to match the $53 \mathrm{keV}$ (standard deviation) calculated energy resolution. A very good agreement is found between the fitted curve and the THM S-factor, as for 18 degrees of freedom, a reduced $\chi^{2}=1.5$ is obtained. The R-matrix astrophysical factor, devoid of effects due to energy resolution, is shown as a red line in figure 1. Finally grey and red bands are used to highlight the uncertainty affecting the R-matrix calculation, for the smeared and unfolded astrophysical factors respectively, taking into account the experimental uncertainties affecting THM data. Table 1 contains the resonance parameters leading to the best fit of the THM data.

To test the effect of the inclusion of the $6417 \mathrm{keV}$ state, R-matrix calculations comprising and excluding such state were implemented. Inserting such a state with the resonance parameters of the literature [20] leads to a reduced $\chi^{2}=3.1$ for 18 degrees of freedom after smearing, two times larger than the calculation completed without its inclusion. This suggests that THM data tend to exclude it from the astrophysical factor. The reason of the increase of the reduced $\chi^{2}$ is also apparent from the inspection of figure 1. The inclusion of the $6417 \mathrm{keV}$ state would cause an increase of the R-matrix S-factor by a factor of more than 3 at $5 \mathrm{keV}$ (from about $300 \mathrm{MeVb}$ up to about 1000 $\mathrm{MeVb}$ ), leading to a discrepancy of $5.5 \sigma$ with respect to the THM astrophysical factor at the same energy. However, more work is necessary to definitely exclude such contribution as our conclusions are presently based on a single point. Yet, it is worth nothing that the THM result agrees with the lack of observation of the mirror state in the ${ }^{15} \mathrm{~N}(\alpha, \alpha){ }^{15} \mathrm{~N}$ cross section [28].

The second important feature is the occurrence of the $6537 \mathrm{keV}$ state reported in $[25,26]$. This level, corresponding to a $126 \mathrm{keV}$ resonance, was not included in the R-matrix calculation by [20]. However, its omission in the present R-matrix calculation would determine an increase in the reduced $\chi^{2}$ to 1.8 . Owing to the THM data energy resolution, the deviation of the calculated S-factor not including the $126 \mathrm{keV}$ resonance from the experimental THM one is well below $5 \sigma$, so a new measurement
Table 1. Parameters of the R-matrix calculation (red line) in figure 1. Resonance energies, corresponding levels in ${ }^{19} \mathrm{Ne}$, spin-parities, $\Gamma_{p}$ and $\Gamma_{\alpha}$ are reported, respectively. For the sub threshold state, the ANC is given instead of $\Gamma_{p}$. A complete discussion on the parameters can be found in [27] and in [20] and references therein.

\begin{tabular}{lllll}
\hline $\mathrm{E}_{\text {res }}(\mathrm{keV})$ & $\mathrm{E}_{x}(\mathrm{keV})$ & $\mathrm{J}^{\pi}$ & $\Gamma_{p}(\mathrm{keV})$ & $\Gamma_{\alpha}(\mathrm{keV})$ \\
\hline-124 & 6286 & $1 / 2^{+}$ & $83.5 \mathrm{fm}^{-1 / 2}$ & 11.6 \\
7 & 6417 & $3 / 2^{-}$ & $1.610^{-41}$ & 0.5 \\
29 & 6440 & $1 / 2^{-}$ & $3.810^{-19}$ & 220 \\
49 & 6460 & $3 / 2^{+}$ & $2.310^{-13}$ & 0.9 \\
126 & 6537 & $7 / 2^{+}$ & $7.110^{-8}$ & 1.5 \\
291 & 6702 & $5 / 2^{+}$ & $2.410^{-5}$ & 1.2 \\
334 & 6745 & $3 / 2^{-}$ & $2.210^{-3}$ & 5.2 \\
665 & 7075 & $3 / 2^{+}$ & 15.2 & 23.8 \\
1461 & 7872 & $1 / 2^{+}$ & 55 & 347 \\
\hline
\end{tabular}

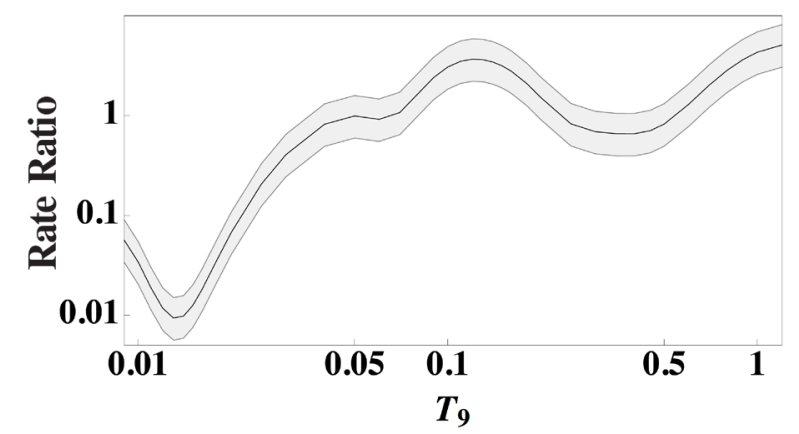

Figure 2. Ratio of the ${ }^{18} \mathrm{~F}(p, \alpha){ }^{15} \mathrm{O}$ reaction rate calculated using the deconvoluted THM S-factor (red band of figure 1) to the one reported in the JINA REACLIB database [30]. Uncertainties of the reaction rate are represented as a grey band.

with improved energy resolution is necessary to clarify its occurrence. This is a very important point since this resonance occurs at astrophysical energies, right in the position where the largest influence from interference between s-wave resonances is expected. Therefore, the presence of the $6537 \mathrm{keV}$ is of significant astrophysical relevance, calling for further indirect high-resolution studies of the ${ }^{18} \mathrm{~F}(\mathrm{p}, \alpha){ }^{15} \mathrm{O}$ reaction using the THM. A more extended discussion on the R-matrix analysis can be found in [27].

\section{Astrophysical consequences}

Using standard equations (see, for instance, [29]), the reaction rate for the ${ }^{18} \mathrm{~F}(\mathrm{p}, \alpha){ }^{15} \mathrm{O}$ reaction was calculated using 
the best-fit R-matrix astrophysical factor, devoid of energy resolution effects (the red band in figure 1). The resulting reaction rate was then divided by the one listed in the JINA REACLIB database [30], for ease of comparison. In fact, as it is shown in [27], the reaction rate is exponentially decreasing with decreasing temperature. The ratio of the THM reaction rate to the REACLIB one is shown in figure 2 as a black line. The REACLIB rate is used for comparison since it is routinely used in novae modeling. The shaded area displays the uncertainty interval corresponding to the red band in figure 1. Temperature on the horizontal axis is given in units of $10^{9} \mathrm{~K}$, namely $\mathrm{T}_{9}=T / 10^{9} \mathrm{~K}$. For $0.1 \lesssim \mathrm{T}_{9} \lesssim 0.5$, typical temperatures of novae nucleosynthesis, the THM reaction rate is about a factor of 2 on the average larger than the one in the REACLIB database [30], in agreement with the results by [20]. The effect of the missing $7 \mathrm{keV}$ resonance causes a decrease of the reaction rate ratio below about $\mathrm{T}_{9} \sim 0.1$, outside the temperature range of astrophysical energies.

The THM reaction rate was then implemented into one-dimensional, hydrodynamic simulations of novae thermonuclear runaways using the SHIVA code [4, 31]. In particular, we tested the influence of the reaction rate for two models of $1.25 \mathrm{M}_{\odot}$ oxygen-neon white dwarfs, accreting $\mathrm{H}$-rich material from the stellar companion at a rate of $2 \times 10^{-10} \mathrm{M}_{\odot} \mathrm{yr}^{-1}$. By changing the ${ }^{18} \mathrm{~F}(\mathrm{p}, \alpha){ }^{15} \mathrm{O}$ reaction rate only, using in one case the THM one and in the other the one listed in REACLIB, we found no change in the dynamical properties of the explosion. Conversely, important differences in the chemical composition of the ejected matter was found, revealing a decrease of the ${ }^{18} \mathrm{~F}$ supply for the THM case by a factor of $\sim 2$. This would imply a reduction of the detectability distance of the $511 \mathrm{keV}$ annihilation line by $\gamma$-ray satellites by a factor $\sim \sqrt{2}$. This may help explaining the present lack of observations of this $\gamma$-ray line.

\section{References}

[1] S. Starrfield, C. Iliadis and W.R. Hix, Classical Novae, 2nd Edition (Cambridge Astrophysics Series. Cambridge, 2006) 77

[2] S. Starrfield, C. Iliadis and W.R. Hix, Publ. Astron. Soc. Pac., 128, 051001 (2016)
[3] J. José and S.N. Shore, Classical Novae, 2nd Edition (Cambridge Astrophysics Series. Cambridge, 2’008) 121

[4] J. José, Stellar Explosions: Hydrodynamics and $\mathrm{Nu}$ cleosynthesis (CRC/Taylor and Francis, Boca Raton, 2016)

[5] M. Hernanz et al., Astrophys. J. 526, L97 (1999)

[6] M. Hernanz and J. José, 5th INTEGRAL Workshop "the INTEGRAL Universe" (ESA SP-552, 2004) 95

[7] M.Dufour, P.Descouvemont, Nucl.Phys. A 785, 381 (2007)

[8] K.E.Rehm et al., Phys.Rev. C 52, R460 (1995)

[9] K.E.Rehm et al., Phys.Rev. C 53, 1950 (1996)

[10] J.-S.Graulich et al., Phys.Rev. C 63, 011302 (2001)

[11] D.W.Bardayan et al., Phys.Rev. C 63, 065802 (2001)

[12] A. St. J. Murphy et al., Phys.Rev. C 79, 058801 (2009)

[13] N. de Sereville et al., Phys.Rev. C 79, 015801 (2009)

[14] D.W.Bardayan et al., Phys.Rev.Lett. 89, 262501 (2002)

[15] C.E.Beer et al., Phys.Rev. C 83, 042801 (2011)

[16] R.L.Kozub et al., Phys.Rev. C 71, 032801 (2005)

[17] M La Cognata et al., Phys.Rev. C 99, 034301 (2019)

[18] J.C.Dalouzy et al., Phys.Rev.Lett. 102, 162503 (2009)

[19] A.S.Adekola et al., Phys.Rev. C 83, 052801 (2011)

[20] D.W.Bardayan et al., Phys.Lett. B 751, 311 (2015)

[21] D.W.Bardayan et al., Phys.Rev. C 96, 055806 (2017)

[22] M. R. Hall et al., Phys. Rev. Lett. 122, 052701 (2019)

[23] D. Kahl et al., Eur. Phys. J. A 55, 4 (2019)

[24] R.E. Tribble et al., Rep. Prog. Phys. 77, 106901 (2014)

[25] S. Cherubini et al., Phys. Rev. C 92, 015805 (2015)

[26] R.G. Pizzone et al., Eur. Phys. J. A 52, 24 (2016)

[27] M. La Cognata et al., Astrophys. J. 846, 65, (2017)

[28] D.W. Bardayan, R.L. Kozub and M.S. Smith, Phys. Rev. C 71, 018801 (2005)

[29] C. Iliadis, Nuclear Physics of Stars (Wiley, New York, 2007)

[30] R.H. Cyburt et al., Astrophys. J. Suppl. Ser. 189, 240 (2010)

[31] J. José and M. Hernanz, Astrophys. J. 494, 680 (1998) 